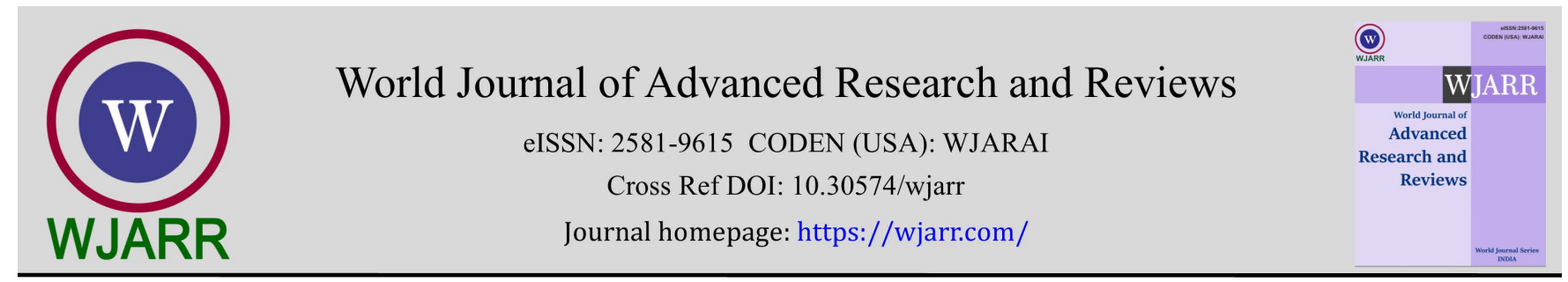

(CASE REPORT)

\title{
Allergic acute coronary syndrome: Amoxicillin/clavulanic acid intake
}

\author{
Filipa Ribeiro Lucas ${ }^{1,}{ }^{*}$, João Gigante ${ }^{2}$, Steve Harakeh ${ }^{3}$ and Pedro Vieira ${ }^{1}$ \\ ${ }^{1}$ Internal Medicine, Unidade Local de Saúde de Castelo Branco (ULSCB), Castelo Branco, Portugal. \\ ${ }^{2}$ Clinical Pathology, Hospital Prof. Doutor Fernando da Fonseca, Amadora, Portugal. \\ ${ }^{3}$ King Fahd Medical Research Center, King Abdulaziz University, Saudi Arabia.
}

World Journal of Advanced Research and Reviews, 2021, 11(02), 064-068

Publication history: Received on 24 June 2021; revised on 02 August 2021; accepted on 04 August 2021

Article DOI: https://doi.org/10.30574/wjarr.2021.11.2.0369

\begin{abstract}
Anaphylaxis rarely manifests as a vasospastic acute coronary syndrome (ACS). Kounis Syndrome (KS) is described as the coincidental occurrence of chest pain and clinical and laboratory findings of ACS. The prognosis depends on the magnitude of the initial allergic response, the patient's sensitivity, comorbidities, the site of antibody antigen reaction, the allergen concentration, and the route of allergen entrance. We report a rare case of KS secondary to antibiotic allergy. This case may suggest that Kounis syndrome should be taught in the differential diagnosis of acute coronary syndromes. Clinicians should be aware of this adverse effect and consider it during diagnostic workup of myocardial injury.
\end{abstract}

Keywords: Allergic acute coronary syndrome; Kounis Syndrome; Amoxicillin-clavulanate; Chest pain; Adverse effect

\section{Introduction}

\subsection{Learning Points}

- Anaphylaxis rarely manifests as a vasospastic acute coronary syndrome with or without the presence of underlying coronary artery disease.

- Kounis Syndrome is described as the coincidental occurrence of chest pain and clinical and laboratory findings of acute coronary syndromes with allergic, hypersensitivity or anaphylactoid reactions. For KS type I, avoiding documented allergen and starting anti-ischemic therapy are the focus of the treatment

- Clinicians should be aware of amoxicillin-clavulanate adverse effects and consider it during diagnostic workup of myocardial injury.

\section{Case description}

The pre-hospital emergency medical service brought a 78-year-old Caucasian female with a medical history of hypertension, atrial fibrillation and OSAS (obstructive sleep apnea and obesity syndrome), to the emergency room (ER) after a sudden loss of consciousness (orthostatic syncope) with absence of prodromal symptoms or of traumatic brain injury. She denied amnesia, sphincter loss or palpitations. She complained of an oppressive chest pain that was resolved upon reaching the ER.

Two months before, she had reported a history of shortness of breath and dyspnea. She also described an 8-day history of productive cough and was treated with oral amoxicillin-clavulanate (875/125 mg) twice per day for 8 days and

\footnotetext{
${ }^{*}$ Corresponding author: Filipa Ribeiro

Internal Medicine, Unidade Local de Saúde de Castelo Branco (ULSCB), Castelo Branco, Portugal.

Copyright (C) 2021 Author(s) retain the copyright of this article. This article is published under the terms of the Creative Commons Attribution Liscense 4.0.
} 
clarithromycin (500 mg) once per day for 5 days. Her family and past medical history did not reveal atopy or frequent allergic conditions. In an out-hospital setting, she was unresponsive with a subsequent drop in the Glasgow Coma Scale (GCS) of 3 and gasping breathing associated with cardiorespiratory arrest. Atropine and epinephrine were administered, with recovery of peripheral perfusion and consciousness and norepinephrine perfusion was also started $(0.33 \mathrm{mcg} / \mathrm{kg} / \mathrm{min})$. Baseline electrocardiogram (ECG) revealed atrial fibrillation and ST elevation in leads I, II, a VF and $\mathrm{T}$ inversion in V1 (Figure 1a). On arrival at the ER (8th antibiotic day), she was responsive with GCS of 15 and normotensive with a blood pressure of $118 / 80 \mathrm{~mm} \mathrm{Hg}$, heart rate of $70 \mathrm{cpm}$. General examination was unremarkable.

This case may suggest that Kounis syndrome should be taught in the differential diagnosis of acute coronary syndromes. Clinicians should be aware of this adverse effect and consider it during diagnostic workup of myocardial injury.

We recorded a control ECG that was completely normal. (Figure 1b). Blood tests have not shown any elevation of inflammatory parameters but revealed elevated levels of cardiac troponin-I $(1.18 \mathrm{mcg} / \mathrm{L}$; maximum of $5 \mathrm{mcg} / \mathrm{L}$ and after on a descending slope). Gas exchange (oxygen of $5 \mathrm{~L} / \mathrm{min}$ ) was within a normal range and with a normal lactate (pH 7.38, pCO2 44 mmHg, p02 126 mmHg, SatO2 100\%, HCO3- $26 \mathrm{mmol} / \mathrm{L}$, lactate $1.8 \mathrm{mmol} / \mathrm{L}$

During hospitalization, the patient also underwent a chest CT scan that identified massive cardiomegaly. Partial atelectasis of the anterior segment of the upper lobe of the right lung, which excluded pulmonary embolism and a Doppler examination of the neck vessels that identified fibrous atheromatous plaques in the bulbar and initial postbulbar segment of the right and left internal carotid arteries, without hemodynamic repercussions. Coronary angiography was performed $24 \mathrm{~h}$ later, revealed $70-90 \%$ smooth extended narrowing in the circumflex artery (Figure 2) and a CT angiography excluded pulmonary embolism. This case was discussed with the Cardiology team and the patient was prescribed aspirin $100 \mathrm{mg} 1$ pill/day and bisoprolol $5 \mathrm{mg} 1$ pill/day. Cardiology exam, revealed that the circumflex artery lesion identified in angiography is not interpreted as the cause of the clinical signs of the patient, suggesting that should be done a myocardial perfusion at rest and pharmacological stress tests.

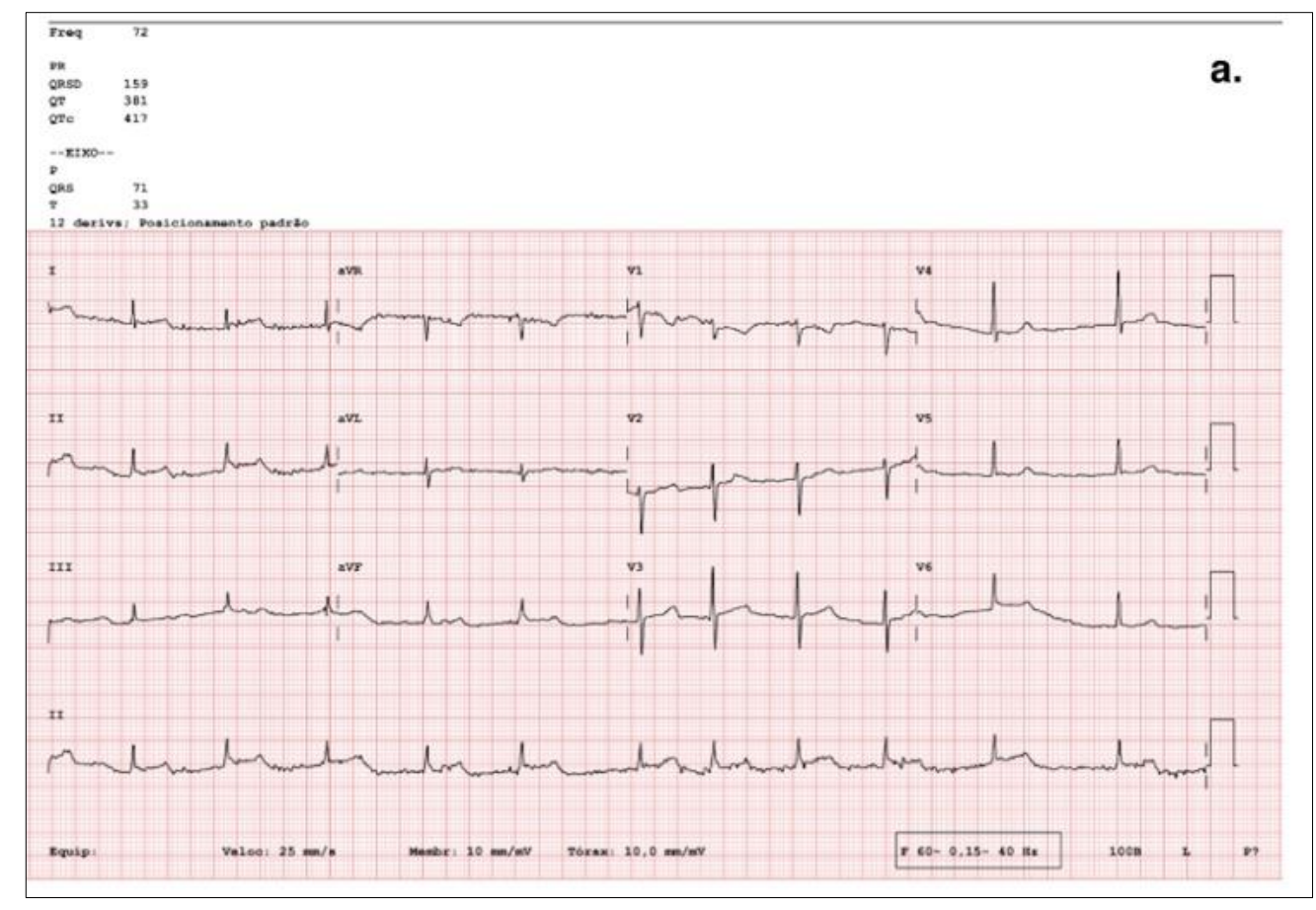

Figure 1 a) Electrocardiogram showed atrial fibrillation, HR of 77cpm and ST elevation in leads I, II, aVF and T inversion in V1

Our patient was discharged after 5 days. From that day on, a myocardial perfusion imaging was performed, showing a normal function, without volume defects or ischemia and with minimum apical perfusion defect. Rest images demonstrated the normal radiotracer distribution, and the patient was discharged by cardiology unit suggestion (Figure 3). She also did allergy tests that confirm this reaction. Work-up, management and ongoing care required multidisciplinary cooperation between the Internal Medicine team, Cardiology and Allergology. Antibiotics that may 
have triggered this condition are contraindicated (amoxicillin, clavulanic acid, and all penicillin-related antibiotics). Our patient is well controlled with anti-ischemic therapy. She is still alive, approximately 1-year post-diagnosis. She is actively followed by internal medicine and cardiology.

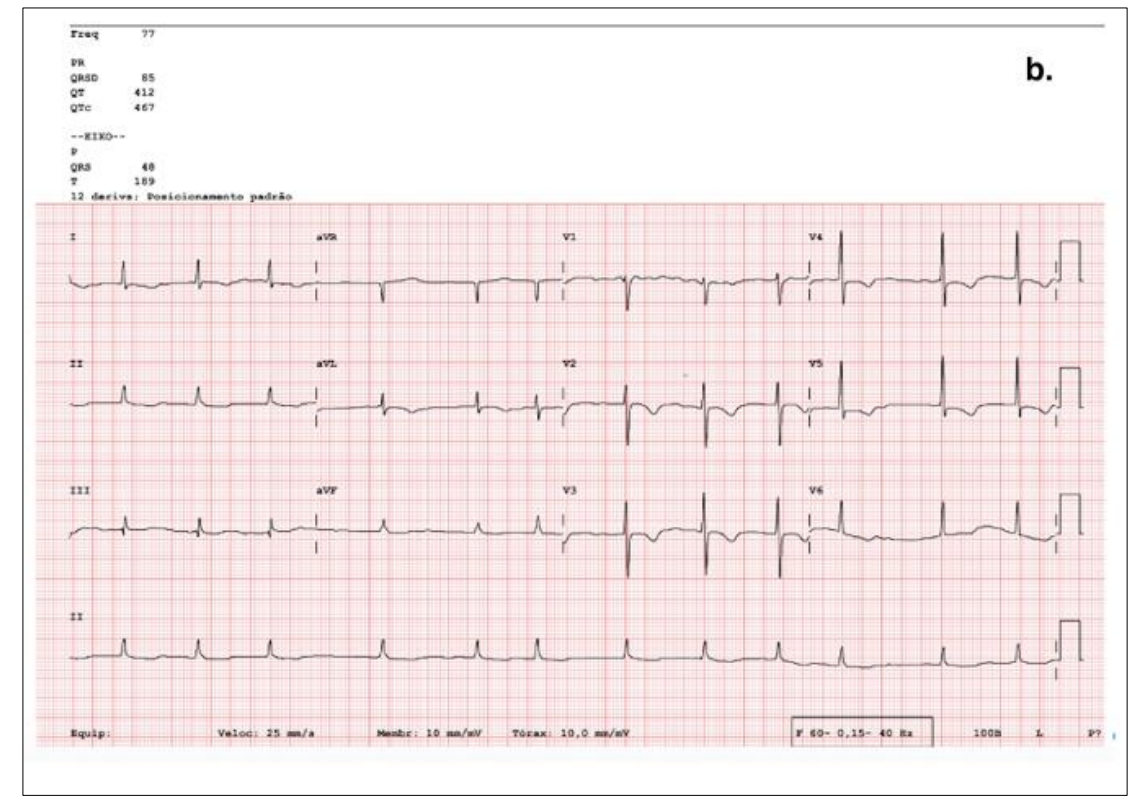

Figure 1 b) Check-up electrocardiogram showed atrial fibrillation, HR of 72cpm, without ischemia defects

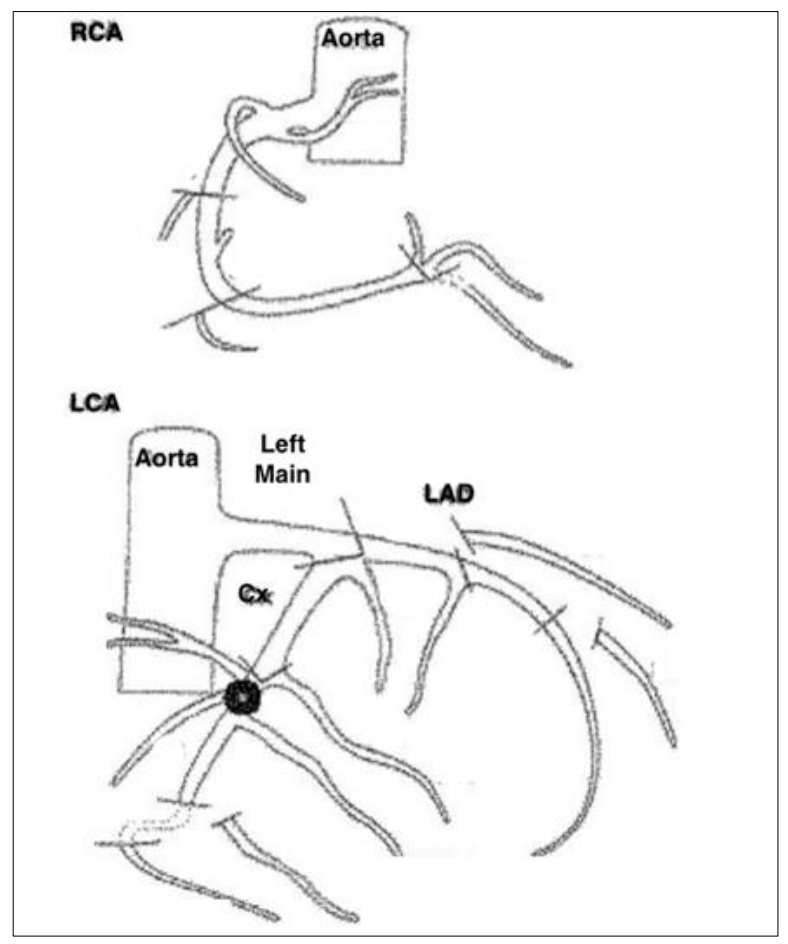

Figure 2 Coronarography showing the distal circumflex of the left coronary artery with stenosis of $70-90 \%$ (represented by the circle). Distal circumflex of the right coronary artery with stenosis of 72-90\%. Common trunk, anterior descending, and right coronary without lesions (Cx - Circumflex; LAD - Left Coronary Artery; LCA - Left

Coronary Artery; RCA - Right Coronary Artery). Report described by the intervention cardiology team. 


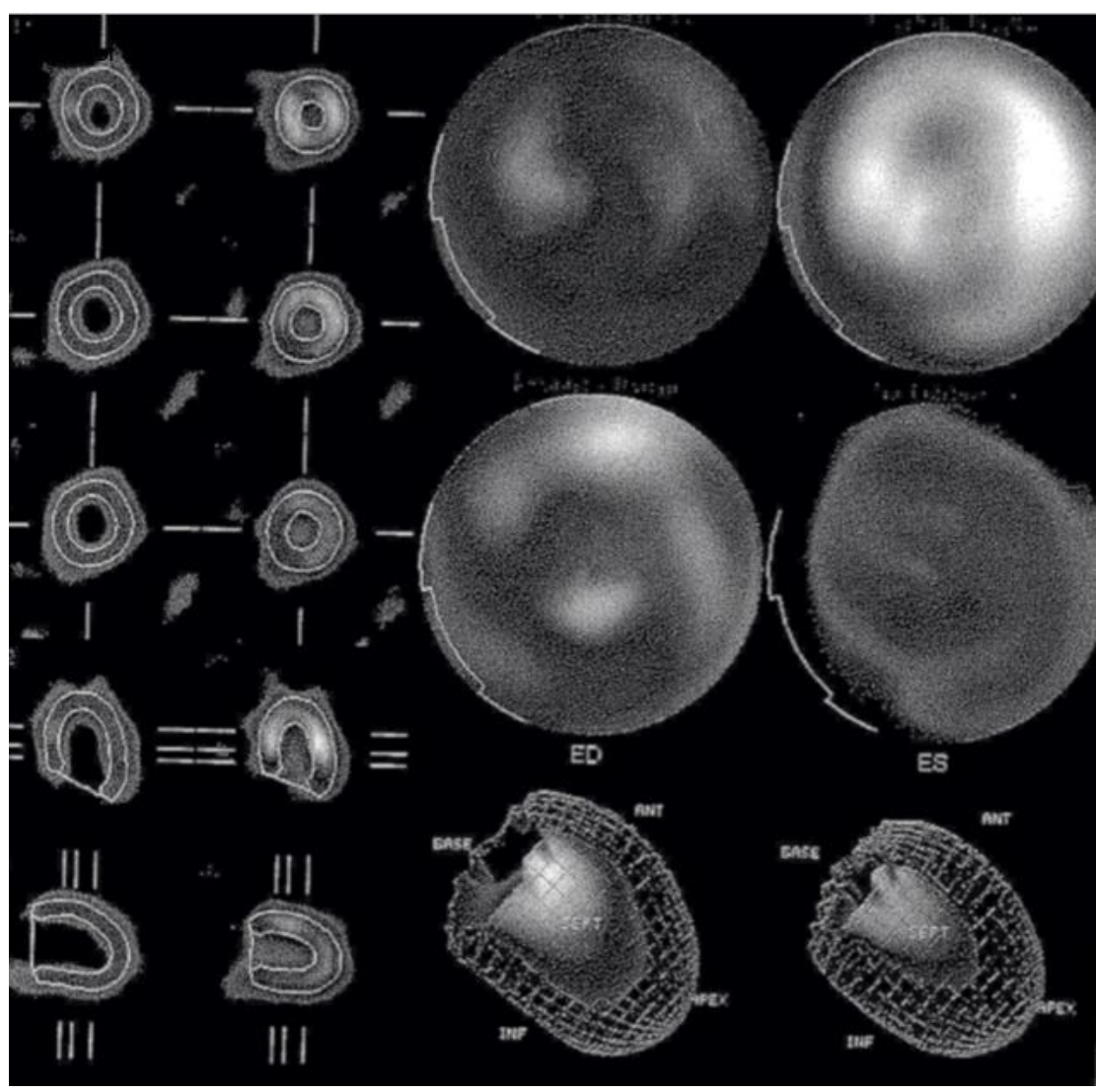

Figure 3 Myocardial scintigraphy showing conserved function and perfusion. Minimal defect in the apical perfusion in the resting study (physiological finding) Report described by the intervention cardiology team.

\section{Discussion}

Following the first report of ACS during an allergic reaction to anti-biotherapy (penicillin) in 1950, its occurrence is rarely described, under-diagnosed and not clearly understood. Myocardial injury and ACS have been also rarely associated with amoxicillin/clavulanic acid intake [1].

Amoxicillin-clavulanate is a widely used broad-spectrum antimicrobial agent. It consists of the semisynthetic penicillin amoxicillin and the beta-lactamase inhibitor, clavulanate potassium [2]. The most frequently reported adverse effects were diarrhea/loose stools, nausea, skin rashes and urticaria. The primary mechanism of its hypersensitivity reaction appears to be the presence of mast cells in heart tissue [3].

Differentiating primary myocardial damage secondary to mast cell activation from the damage caused by global myocardial hypoperfusion can be challenging [4]. For example, tachycardia, ventricular dysfunction, and atrioventricular block are some of the effects of KS mediators. Anaphylaxis can also cause systemic vasodilation that decreases venous return and, consequently, cardiac output, resulting in prolonged hypotension that may precipitate a reduction in coronary flow ending in myocardial ischemia that remains an alternative potential explanation for the allergic acute coronary syndrome. Therefore, the variability on the underlying pathogenesis produces a wide clinical spectrum.

KS has 3 variants described. The first is seen in patients without previous heart disease, in whom the inflammatory cascade causes coronary vasospasm accompanied by elevated levels of myocardial damage markers. The second is observed in patients with pre-existing atherosclerotic disease, resulting in rupture of the atherosclerotic plaque and the third is caused by stent thrombosis [5]. 


\section{Conclusion}

It is important to appropriately recognize and treat KS in patients with exposure to a documented allergen which requires the thoughtful use of several common drugs. The primary focus of treatment should be directed towards the allergic insult and removal of the offending allergens.

In previous reports, only a few amoxicillin-clavulanate-induced KS cases were reported in patients who had normal coronary arteries. This case report is a KS type I, in which stopping amoxicillin-clavulanate and starting an anti-ischemic treatment were the best options. We believe that the signs and symptoms of our patient completely resolved after withdrawal of the drug and aspirin intake. Furthermore, other etiologies of ACS were appropriately ruled out by angiography and myocardial perfusion imaging. This case may suggest that Kounis syndrome should be taught in the differential diagnosis of acute coronary syndromes. Clinicians should be aware of this adverse effect and consider it during diagnostic workup of myocardial injury.

\section{Compliance with ethical standards}

\section{Acknowledgments}

The researchers appreciated all the corrections suggested and recommended by the experts before field testing. This research received no precise fund from any agency in public or private sectors.

\section{References}

[1] Ralapanawa D, Kularatne S. Kounis syndrome secondary to amoxicillin/clavulanic acid administration: a case report and review of literature. BMC research notes. 2015; 8(1): 1-6.

[2] Tavil Y, Turfan M, Türkoğlu S, Abacı A. Kounis syndrome secondary to amoxicillin/clavulanic acid use. International journal of cardiology. 2008; 124(1): e4-e7.

[3] Galli SJ, Kalesnikoff J, Grimbaldeston MA, Piliponsky AM, Williams CM, Tsai M. Mast cells as "tunable" effector and immunoregulatory cells: recent advances. Annu Rev Immunol. 2005; 23: 749-86.

[4] Akyel A, Murat SN, Çay S, Kurtul A, Öcek AH, Cankurt T. Late drug eluting stent thrombosis due to acemetacine: type III Kounis syndrome: Kounis syndrome due to acemetacine. International journal of cardiology. 2012; 155(3): 461-2.

[5] Nikolaidis LA, Kounis NG, Gradman AH. Allergic angina and allergic myocardial infarction: a new twist on an old syndrome. The Canadian journal of cardiology. 2002; 18(5): 508-11. 